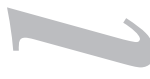

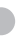
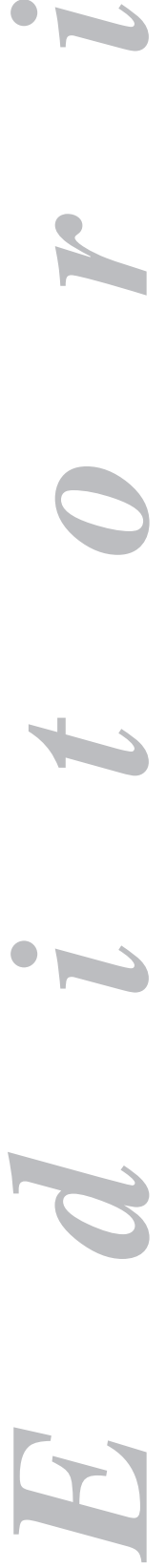

\section{Gastro-esophageal reflux disease and irritable bowel syndrome - why are they associated?}

Gastroesophageal reflux disease (GERD) and irritable bowel syndrome (IBS) are the most prevalent gastrointestinal disorders in the general population. In Spain around $10 \%$ of the population have typical reflux symptoms for at least one day a week, and $20 \%$ have them on an occasional basis (1); incidence is around $4 \%$ annually (2), whereas the prevalence of IBS is around $2-12 \%$ (3) depending on the defining criteria. Moreover, around $28 \%$ of people with GERD symptoms (4) and two thirds of people with IBS (5) consult a doctor each year. From a worldwide perspective both GERD (6) and IBS (7) exhibit similarly high prevalence scores, even though regional differences exist.

Given the high prevalence of both diseases among the population their common association is hardly surprising; in fact, patients with heartburn as well as abdominal pain and bowel transit changes are common in our practices. To date we have considered these patients as carriers of two unrelated diseases, with the diagnostic and therapeutic complications this entails. However, population-based studies show that the association of GERD and IBS does occur much more commonly than would be expected by random causes (8). A recent systematic review of the literature suggests that the prevalence of GERD in subjects with IBS is 30\%, much higher than the $19 \%$ seen in the general population, whereas the prevalence of IBS in individuals with GERD is $60 \%$, notably higher than $12 \%$ of the population (9). This fact suggests a number of questions, most of them as yet unanswered: should we still consider them two unrelated diseases? Which factor may explain the above-mentioned association? Has this association any diagnostic or therapeutic implications?

While it has been suggested that IBS symptoms may be a component of GERD's clinical expression (10), based on the improvement of abdominal symptoms in patients diagnosed with GERD on antireflux therapy, most of the available scientific evidence suggests that these are separate disorders sharing some common factor.

The search for this shared factor is a scientific challenge with dramatic diagnostic and therapeutic implications; however, it has just begun and several years will probably go by until data apt to be translated into clinical practice will be available.

Major factors explored include genetic and psychological aspects, motility disorders, and sensitivity changes. Lembo et al. (11) have shown, in a study with twins, that genetic contributions to GERD and IBS development are different; while this is only a study to be subsequently confirmed by further research, individual studies performed to explore susceptibility to GERD or IBS also suggest no shared genes. A gastrointestinal tract smooth muscle disorder has been suggested to support this association, but data available thus far are scarcely convincing (12). Some studies suggest that a shared factor could be visceral hypersensitivity, and generalized (nonorgan-specific) hypersensitivity has been found in functional GI disorders (13) 
while esophageal hypersensitivity was seen in patients with IBS (14). Finally, psychological factors play a relevant role both in $\operatorname{GERD}(2,15)$ and IBS $(16)$, and population-based studies have been suggested for potential explanations (17); however, at least from a clinical point of view, these do not seem determinant in the association of both disorders (15).

In this issue of Revista Española de Enfermedades Digestivas, Max Schmulson et al. (18) report novel data on this search for factors, and focus on the hypothesis that obesity may explain it. Obesity is a Western world epidemics $(19,20)$; its prevalence progressively increased during the last few decades, is clearly associated with GERD (21), and is linked to abdominal symptoms such as diarrhea and distension (22). Using adequate methods, their results reinforce both the GERD and IBS superposition, and an association between obesity and reflux symptoms, but also clearly show that obesity provides no explanation why GERD and IBS are associated more commonly than expected by chance. Even a negative result detracts no value from this report, particularly bearing in mind that we just set out in our way to explain something that is extremely common in our practices on a daily basis.

\author{
E. Rey and M. García-Arredondo \\ Department of Digestive Diseases. Hospital Clínico San Carlos. Universidad \\ Complutense. Madrid, Spain
}

\title{
REFERENCES
}

1. Diaz-Rubio M, Moreno-Elola-Olaso C, Rey E, Locke GR, III, Rodriguez-Artalejo F. Symptoms of gastro-oesophageal reflux: prevalence, severity, duration and associated factors in a Spanish population. Aliment Pharmacol Ther 2004; 19: 95-105.

2. Rey E, Alvarez-Sanchez A, Rodriguez-Artalejo F, Moreno Elola-Olaso C, Almansa C, Diaz-Rubio M. Onset and disappearance rates of gastroesophageal reflux symptoms in the Spanish population, and their impact on quality of life. Rev Esp Enferm Dig 2009; 101: 477-82.

3. Mearin F, Badia X, Balboa A, Baro E, Caldwell E, Cucala M, et al. Irritable bowel syndrome prevalence varies enormously depending on the employed diagnostic criteria: comparison of Rome II versus previous criteria in a general population. Scand J Gastroenterol 2001; 36: 1155-61.

4. Rey E, Moreno-Elola-Olaso C, Rodriguez-Artalejo F, Diaz-Rubio M. Medical consultation for gastro-oesophageal reflux symptoms: reasons and associated factors. Digestion 2004; 70: 173-7.

5. Badia X, Mearin F, Balboa A, Baro E, Caldwell E, Cucala M, et al. Burden of illness in irritable bowel syndrome comparing Rome I and Rome II criteria. Pharmacoeconomics 2002; 20: 749-58.

6. Dent J, El-Serag HB, Wallander MA, Johansson S. Epidemiology of gastro-oesophageal reflux disease: a systematic review. Gut 2005; 54: 710-7.

7. Rey E, Talley NJ. Irritable bowel syndrome: novel views on the epidemiology and potential risk factors. Dig Liver Dis 2009; 41: 772-80.

8. Jung HK, Halder S, McNally M, Locke GR, III, Schleck CD, Zinsmeister AR, et al. Overlap of gastro-oesophageal reflux disease and irritable bowel syndrome: prevalence and risk factors in the general population. Aliment Pharmacol Ther 2007; 26: 453-61.

9. Nastaskin I, Mehdikhani E, Conklin J, Park S, Pimentel M. Studying the overlap between IBS and GERD: a systematic review of the literature. Dig Dis Sci 2006; 51: 2113-20.

10. Gasiorowska A, Poh CH, Fass R. Gastroesophageal reflux disease (GERD) and irritable bowel syndrome (IBS)--is it one disease or an overlap of two disorders? Dig Dis Sci 2009; 54: 1829-34.

11. Lembo A, Zaman M, Jones M, Talley NJ. Influence of genetics on irritable bowel syndrome, gastro-oesophageal reflux and dyspepsia: a twin study. Aliment Pharmacol Ther 2007; 25: 1343-50.

12. Smart HL, Nicholson DA, Atkinson M. Gastro-oesophageal reflux in the irritable bowel syndrome. Gut 1986; 27: 1127-31

13. Trimble KC, Farouk R, Pryde A, Douglas S, Heading RC. Heightened visceral sensation in functional gastrointestinal disease is not site-specific. Evidence for a generalized disorder of gut sensitivity. Dig Dis Sci 1995; 40: 1607-13.

14. Costantini M, Sturniolo GC, Zaninotto G, D'Inca R, Polo R, Naccarato R, et al. Altered esophageal pain threshold in irritable bowel syndrome. Dig Dis Sci 1993; 38: 206-12. 
15. Rey E, Garcia-Alonso M, Moreno-Ortega M, Almansa C, Alvarez-Sanchez A, Diaz-Rubio M. Influence of psychological distress on characteristics of symptoms in patients with GERD: the role of IBS comorbidity. Dig Dis Sci 2009; 54: 321-7.

16. Rey E, Moreno OM, Garcia Alonso MO, Diaz-Rubio M. Constructive thinking, rational intelligence and irritable bowel syndrome. World J Gastroenterol 2009; 15: 3106-13.

17. Lee SY, Lee KJ, Kim SJ, Cho SW. Prevalence and risk factors for overlaps between gastroesophageal reflux disease, dyspepsia, and irritable bowel syndrome: a population-based study. Digestion 2009; 79: 196-201.

18. Schmulson M, Pulido D, Escobar C, Farfan-Labone B, Gutierrez-Reyes G, Lopez-Alvarenga JC. Heartburn and other related symptoms are independent of body mass index in IBS. Rev Esp Enferm Dig 2010; 102: 229-33.

19. Wang Y, Beydoun MA. The obesity epidemic in the United States -gender, age, socioeconomic, racial/ethnic, and geographic characteristics: a systematic review and meta-regression analysis. Epidemiol Rev 2007; 29: 6-28.

20. Schroder H, Elosua R, Vila J, Marti H, Covas MI, Marrugat J. Secular trends of obesity and cardiovascular risk factors in a Mediterranean population. Obesity (Silver Spring) 2007; 15: 557-62.

21. Corley DA, Kubo A. Body mass index and gastroesophageal reflux disease: a systematic review and metaanalysis. Am J Gastroenterol 2006; 101: 2619-28.

22. Delgado-Aros S, Locke GR, III, Camilleri M, Talley NJ, Fett S, Zinsmeister AR, et al. Obesity is associated with increased risk of gastrointestinal symptoms: a population-based study. Am J Gastroenterol 2004; 99: 1801-6.

\section{Enfermedad por reflujo gastroesofágico y síndrome de intestino irritable, ¿por qué se asocian?}

La enfermedad por reflujo gastroesofágico (ERGE) y el síndrome de intestino irritable (SII) son las enfermedades digestivas más prevalentes en la población general. En España, un 10\% de la población sufre síntomas típicos de reflujo al menos un día a la semana y un $20 \%$ los sufre de forma ocasional (1), con una incidencia en torno al $4 \%$ anual (2), mientras la prevalencia de SII se sitúa en un 2-12\% (3), dependiendo de los criterios que se utilicen para definirla. Más aún, en torno al $28 \%$ de las personas con síntomas de ERGE (4) y las dos terceras partes de personas con síntomas de SII (5) consultan al médico cada año. Si lo miramos desde la perspectiva mundial, tanto la ERGE (6) como el SII (7) muestran unas cifras de prevalencia igualmente elevadas, aunque con ciertas diferencias regionales.

Dada la alta prevalencia de ambas enfermedades en la población, no es de extrañar que se asocien con frecuencia y, de hecho, en nuestras consultas, es habitual el paciente con pirosis que además refiere dolor abdominal y alteraciones del tránsito. Hasta ahora, hemos considerado al paciente como portador de dos enfermedades independientes, con las implicaciones diagnósticas y terapéuticas que ello conlleva. Sin embargo, estudios poblacionales muestran que la asociación de ERGE y SII ocurre con una frecuencia mucho mayor de la cabría esperar por el mero azar (8). Una reciente revisión sistemática de la literatura señala que la prevalencia de ERGE en personas con SII es del $30 \%$, muy superior al 19\% de la población general, mientras la prevalencia de SII en personas con ERGE es del 60\%, notablemente superior 
al $12 \%$ de la población (9). Este hecho sugiere muchas preguntas, la mayoría aun sin contestar: ¿debemos seguir considerándolas dos enfermedades completamente diferentes?, ¿cuál es el factor que explica dicha asociación?, ¿tiene alguna implicación diagnóstica o terapéutica dicha asociación?

Aunque se han sugerido los síntomas del SII podrían ser un componente más de la expresión clínica de la ERGE (10), basándose en una mejoría de los síntomas abdominales en pacientes diagnosticados de ERGE que han recibido tratamiento antirreflujo, la mayor parte de la información científica disponible sugiere que son dos trastornos distintos que comparten algún factor común.

La búsqueda de este factor común es un reto científico cuyo resultado podría tener unas enormes implicaciones diagnósticas y terapéuticas; sin embargo, no ha hecho más que empezar y probablemente pasarán algunos años hasta que tengamos una información trasladable a nuestra práctica clínica.

Entre los grandes factores que se han explorado encontramos factores genéticos, psicológicos, alteraciones de la motilidad y alteraciones de la sensibilidad. Lembo y cols. (11) han mostrado, en un estudio de gemelos, que la contribución genética para el desarrollo de la ERGE y el SII es diferente; aunque es tan sólo un estudio y debe confirmarse en estudios posteriores, tampoco los estudios que individualmente se han realizado para estudiar la susceptibilidad para padecer ERGE o SII sugiere ningún gen común. Se ha señalado que detrás de esta asociación podría existir un trastorno de la musculatura lisa de todo el tracto gastrointestinal, sin embargo, los datos hasta ahora disponibles no son demasiado convincentes (12). Algunos estudios apuntan que el factor común podría ser la hipersensibilidad visceral, habiéndose observado una hipersensibilidad generalizada (no órgano específica) en los trastornos funcionales digestivos (13) y una hipersensibilidad esofágica en pacientes con SII (14). Finalmente, los factores psicológicos tienen un peso importante tanto en la ERGE $(2,15)$ como en el SII $(16)$ y se han señalado en estudios poblacionales como una posible explicación (17), pero al menos desde el punto de vista clínico no parece que sean determinantes en la asociación de ambos trastornos (15).

En este número de la Revista Española de Enfermedades Digestivas, Max Schmulson y cols. (18) nos ofrecen nuevos datos en esta búsqueda de factores, centrándose en la hipótesis de que la obesidad pudiera explicarla. La obesidad es una epidemia en el mundo occidental $(19,20)$, con un aumento progresivo de su prevalencia en las últimas décadas, que está claramente relacionada con la ERGE (21) y se asocia con algunos síntomas abdominales, como la diarrea y la distensión (22). Usando una metodología adecuada, sus resultados reafirman tanto la superposición entre ERGE y SII como la asociación entre obesidad y síntomas de reflujo, pero claramente muestran que la obesidad no explica por qué la ERGE y el SII se asocian con mayor frecuencia de lo esperado por azar. Aunque el resultado sea negativo no es por ello menos valorable, especialmente teniendo en cuenta que no hemos hecho sino empezar el camino para explicar algo que ocurre en nuestra consulta todos los días con extraordinaria frecuencia.

E. Rey y M. García-Arredondo

Servicio de Aparato Digestivo. Hospital Clínico San Carlos. Universidad Complutense. Madrid 